\title{
ANÁLISIS DE LA DISCAPACIDAD SEVERA EN CHILE EN PACIENTES ATENDIDOS EN LA ATENCIÓN PRIMARIA, \\ AÑO 2011-2012
}

ANALYSIS OF DISABILITY SEVERE CHILE IN PATIENTS TREATED IN PRIMARY CARE, 2011-2012

\section{Johnny Acevedo A. ${ }^{1}$, Ivonne Alhers M. ${ }^{2}$, Oscar Henríquez T. ${ }^{3}$, Hernán Aguilera M. ${ }^{4}$}

\begin{abstract}
Resumen:
Chile enfrenta un envejecimiento acelerado de su población que obliga a estudiar este fenómeno y adecuar la política pública en salud. El presente es un estudio descriptivo de corte transversal, cuyo objetivo es analizar datos relevantes de los pacientes con discapacidad severa en Chile. La fuente fue el Departamento Estadístico e Informático en Salud del Ministerio de Salud de Chile, logrando caracterizar esta población, identificar la cobertura nacional y por Servicios de Salud, analizar prevalencias de los pacientes bajo control, la capacidad de oferta y el impacto de la política. Se encontró que existe 1 paciente con discapacidad severa por cada 100 beneficiarios de FONASA el año 2011, lo que aumentó a 2,3 el año 2012. El promedio de pacientes bajo control fue de 931 el año 2011 y 1.079 el 2012. Los pacientes se concentran en las zonas del centro y sur del país, más del 60\% son mujeres, excepto en los SS de Aysén, Araucanía Norte y Magallanes. Por grupos de edad, el $72 \%$ de estos pacientes son mayores de 65 años, no existiendo diferencias importantes por Servicios de Salud (SS) en ambos años. Las tasas de discapacidad severa por cada 1.000 beneficiarios de FONASA son más altas el año 2012 respecto del 2011; lo que aumenta con la edad, especialmente en los mayores de 65 años. Lo mismo ocurre según sexo y SS. Las tasas más altas se concentran en la zona sur del país. Para el análisis de los datos se usó el programa Excel.
\end{abstract}

\section{Palabras Clave:}

Dependencia Severa, perfil epidemiológico y sociodemográfico, DEIS, MINSAL.

\begin{abstract}
:
Chile faces a rapidly aging population requiring study this phenomenon and adapt public health policy. This is a descriptive crosssectional study, which aims to analyze relevant data from patients with severe disabilities in Chile. The source was the Statistical and Computer Department for Health, Ministry of Health of Chile, being able to characterize this population, identify national coverage and health services, analyze prevalence of patients under control, supply capacity and the impact of political. There is 1 patient with severe disabilities FONASA 100 beneficiaries in 2011, which increased to 2.3 by 2012. The average patient under control was 931 in 2011 and 1,079 in 2012. Patients are concentrated in the center of the south, most areas 60\% are women, except SS Aysen, Magallanes and Araucanía Norte. By age group, $72 \%$ of these patients are over 65, there being no significant differences for Health Services (SS) in both years. Severe disability rates per 1,000 beneficiaries FONASA are higher in 2012 compared to 2011; which increases with age, especially over 65 years. The same goes according to sex and SS. The highest rates are concentrated in the southern region. For data analysis the Excel program was used
\end{abstract}

\section{Keywords:}

Severe Dependency, epidemiological and socio-demographic profile, DEIS, MINSAL.

1 Matrón, Magíster en Salud Pública, Magíster en Administración y Dirección de Empresas, Profesor Adjunto Depto. Atención Primaria y Salud Familiar, Facultad de Medicina Universidad de Chile. jhonnyacevedo@med.uchile.cl

2 Enfermera, Diplomada en Desarrollo Organizacional, Directora del Depto. Atención Primaria y Salud Familiar, Profesora Asistente, Facultad de Medicina Universidad de Chile. ialhers@med.uchile.cl

3 Dr. Oscar Henríquez, Médico de Familia, Profesor Asistente, Depto. Atención Primaria y Salud Familiar, Facultad de Medicina Universidad de Chile. ohenriquez@med.uchile.cl

4 Matrón, Magíster en Salud Pública, Profesor asistente Depto. Atención Primaria y Salud Familiar, Facultad de Medicina Universidad de Chile. hernanaguilera@med.uchile.cl 


\section{INTRODUCCIÓN}

Chile se encuentra en una fase de transición demográfica avanzada, fase IV (BID/CEPAL/CELADE, 2000), donde todos los informes nacionales e internacionales muestran cambios importantes en indicadores sanitarios asociados a una disminución franca de mortalidad infantil, de las tasas de fecundidad, mantención de las tasas de mortalidad general, y disminución de las tasas de crecimiento vegetativo, proyectándose para el 2050 que la población menor de 4 años sea numéricamente inferior a los mayores de 80 años.

Se entiende por dependencia a la disminución o ausencia de la capacidad para realizar alguna actividad dentro de un rango de normalidad (Dorentes G. et col., 2001), con una incapacidad funcional para realizar actividades de la vida diaria (Barthel D., Mahoney F., 1965), existiendo la necesidad de ser ayudado para poder interactuar con el medio ambiente (Baltes M., Wahl $\mathrm{H} ., 1990)$, requiriendo apoyo para desenvolverse normalmente frente a diversas áreas adaptativas (IMERSO, 1997), requiriendo de terceros para estas actividades de la vida diaria (Abanto A., 2000) y donde estas personas con dependencia física, psíquica e intelectual son incapaces de llevar una vida independiente de manera efectiva (Clasificación Internacional de Deficiencias, Discapacidades y Minusvalías, 1980). Para la Organización Mundial de la Salud (OMS) la Independencia Física es la capacidad del sujeto para llevar habitualmente una existencia independiente efectiva (Querejeta M., 2004).

Un hito importante a nivel nacional es la publicación el año 2002 de la Ley N 19.828 que crea el Servicio Nacional del Adulto Mayor, que entre otras cosas clasifica a este grupo como todos aquellos habitantes mayores de 60 años en Chile (Observatorio Demográfico parea América Latina y el Caribe, 2012) (INE, 2008). A la vez, la esperanza de vida en el país ha aumentado de 63,6 a 78,4 años de vida desde el quinquenio 1970-1975 a 2005-2010, representando un aumento porcentual de $18,8 \%$, proyectándose que este aumento llegue a 22,5\% al año 2050. La población mayor de 60 años en Chile representaba al año 2010 un 13\% y se proyecta que este mismo grupo etario alcance a un $31 \%$ de la población general el año 2050 . El índice de dependencia ha caído de $73 \%$ el año 1970 a $26 \%$ el año 2010, proyectándose un nuevo aumento a $61 \%$ el año 2050. El Índice de envejecimiento del país representaba un 24,7\% el año 1980, un 59,1\% el año 2010 y se proyecta aumente a $132 \%$ el año 2030.
En Chile el 24,1\% de las personas de 60 años o más que tienen algún nivel de dependencia, sea esta leve, moderada o severa (Calvo A., Tartakowsky A., Maffei T., 2001). Según el Estudio Nacional de la Dependencia en las Personas Mayores del año 2010 (SENAMA, 2010), la limitación funcional severa incluye a individuos postrados que están confinados a su cama, sujetos que presenten demencia de cualquier grado con puntaje MMSE corto $<13$ puntos y puntaje test de actividades funcionales de Pffefer $>5$ puntos (Quiroga P., Albala C., Klaasen G., 2004), presencia de incapacidad para efectuar 1 Actividad Básica de la Vida Diaria ${ }^{5}$ (ABVD) (Katz S., et col., 1964) con la excepción de bañarse o incapacidad para efectuar 2 Actividades Instrumentales de la Vida Diaria ${ }^{6}$ (AIVD) (Lawton M., Brody E., 1969). Las personas que cumplen con alguno de los 3 criterios mencionados, son consideradas como personas dependientes severas.

La discapacidad severa cobra mayor notoriedad con el inicio del Gobierno de la Presidenta Bachelet el año 2006, donde la medida 6 b de su programa de gobierno es una de las 36 medidas definidas a realizarse dentro de los 100 primeros días de su gestión, lo que además de prevenir la pérdida de funcionalidad temprana pone el énfasis en la rehabilitación con enfoque comunitario del adulto mayor postrado con pérdida de funcionalidad, iniciando además el apoyo a familias cuidadoras de estos pacientes (Norma de cuidados domiciliarios de personas que sufren Discapacidad Severa, MINSAL, 2006). Lo anterior se refuerza con la publicación de la Ley $N^{\circ} 20.422$ del 10 de febrero de 2010, donde se pone énfasis al derecho de igualdad de oportunidades de las personas con discapacidad, con énfasis en la inclusión social y la no discriminación. También se aseguran las acciones preventivas y recuperativas como responsabilidad del Estado, independiente del tipo de discapacidad de las personas (Biblioteca del Congreso Nacional de Chile, 2010).

\footnotetext{
Katz y colaboradores definieron como actividades de la vida diaria el bañarse, vestirse, caminar, comer, usar el excusado, acostarse y levantarse de la cama.

6 Lawton y Brody definieron como actividades instrumentales de la vida diaria el preparar comida, manejar su propio dinero, salir solo de su casa, efectuar compras, hacer o recibir llamadas telefónicas, efectuar quehaceres livianos de la casa, organizar y tomar sus propios medicamentos.
} 


\section{MÉTOdo}

El presente es un estudio descriptivo de corte transversal, que surge como una necesidad investigativa de los autores por conocer cómo se está desarrollando el enfoque asistencial de los pacientes con discapacidad en el país, en tanto es un problema de salud en aumento producto de los cambios sociales, de los determinantes que influyen en la enfermedad, del envejecimiento poblacional, así como porque empíricamente existe la percepción de un déficit de la política pública sanitaria para una óptima asistencia a estos pacientes, tanto a nivel de la Atención Primaria en Salud como a nivel Hospitalario. Frente a la complejidad de acceso a los datos a nivel país por Servicios de Salud, se decide analizar los datos de acceso público existentes en el Departamento Estadístico e Informático en Salud (DEIS) del Ministerio de Salud de Chile (MINSAL). Cabe señalar que el MINSAL tiene registro de datos de pacientes con discapacidad severa desde el año 2006 en adelante, sin embargo son datos distintos según necesidad anual del programa y por lo tanto no permiten una comparación seriada por año, salvo los analizados en este estudio. De acuerdo a lo anterior, se trabajó en el análisis de estos datos correspondientes a los años 2011 y 2012, únicos datos comparables a nivel nacional y por Servicio de Salud (SS) según esta fuente. Los datos fueron exportados a Excel para los cruces respectivos, así como para la construcción de tablas y gráficos según años, sexo, SS y país.

\section{Resultados}

TABLA N ${ }^{\circ} 1$

Distribución numérica y porcentual de la Discapacidad Severa bajo control Según SS y País, años 2011 - 2012.

\begin{tabular}{|c|c|c|c|c|}
\hline & 2011 & & 2012 & \\
\hline & $\mathrm{N}^{\circ}$ & $\%$ & $\mathrm{~N}^{\circ}$ & $\%$ \\
\hline País & 26.054 & & 30198 & \\
\hline Arica & 255 & 0,98 & 344 & 1,14 \\
\hline Iquique & 268 & 1,03 & 366 & 1,21 \\
\hline Antofagasta & 384 & 1,47 & 703 & 2,33 \\
\hline Atacama & 270 & 1,04 & 143 & 0,47 \\
\hline Coquimbo & 1.381 & 5,30 & 1296 & 4,29 \\
\hline Valparaíso San Antonio & 1.160 & 4,45 & 1090 & 3,61 \\
\hline Viña del Mar Quillota & 1.366 & 5,24 & 1176 & 3,89 \\
\hline Aconcagua & 313 & 1,20 & 422 & 1,40 \\
\hline Metropolitano Norte & 722 & 2,77 & 1109 & 3,67 \\
\hline Metropolitano Occidente & 1.488 & 5,71 & 1756 & 5,81 \\
\hline Metropolitano Central & 1.010 & 3,88 & 1205 & 3,99 \\
\hline Metropolitano Oriente & 1.400 & 5,37 & 2031 & 6,73 \\
\hline Metropolitano Sur & 2.366 & 9,08 & 2340 & 7,75 \\
\hline Metropolitano Sur Oriente & 1.114 & 4,28 & 1454 & 4,81 \\
\hline Del Libertador B. O’Higgins & 815 & 3,13 & 1608 & 5,32 \\
\hline
\end{tabular}




\begin{tabular}{|c|c|c|c|c|}
\hline & 2011 & & 2012 & \\
\hline Del Maule & 1.333 & 5,12 & 1888 & 6,25 \\
\hline$\tilde{N}$ uble & 1.623 & 6,23 & 1419 & 4,70 \\
\hline Concepción & 1.251 & 4,80 & 1384 & 4,58 \\
\hline Arauco & 340 & 1,30 & 258 & 0,85 \\
\hline Talcahuano & 697 & 2,68 & 710 & 2,35 \\
\hline Biobío & 1.107 & 4,25 & 1127 & 3,73 \\
\hline Araucanía Norte & 409 & 1,57 & 528 & 1,75 \\
\hline Araucanía Sur & 2.028 & 7,78 & 2271 & 7,52 \\
\hline Valdivia & 749 & 2,87 & 932 & 3,09 \\
\hline Osorno & 478 & 1,83 & 590 & 1,95 \\
\hline Del Reloncaví & 935 & 3,59 & 1163 & 3,85 \\
\hline Chiloé & 477 & 1,83 & 602 & 1,99 \\
\hline Aisén & 60 & 0,23 & 100 & 0,33 \\
\hline Magallanes & 255 & 0,98 & 183 & 0,61 \\
\hline
\end{tabular}

Fuente: DEIS - MINSAL, Chile.

Entre el año 2011 a 2012 hubo un aumento porcentual de $13 \%$ en el bajo control de pacientes con discapacidad severa. Las importancias relativas por año y SS, son similares, concentrándose en los SS Metropolitano Sur, Araucanía Sur, Ñuble, Metropolitano Oriente, Coquimbo y Viña del Mar Quillota las más altas, en tanto en las regiones extremas se observan las más bajas. El promedio nacional de pacientes con DS bajo control fue de 907 para el año 2011 y 1.079 el 2012, observándose que el $50 \%$ de los Servicios de Salud están por sobre el promedio el 2011 y $57 \%$ el 2012. De acuerdo a estos datos y al hacer un cruce con número de beneficiarios adscritos al seguro público del Fondo Nacional de Servicio de Salud (FONASA, 2012) para los años 2011 y 2012, teníamos 1,97 pacientes con discapacidad severa por cada 1.000 beneficiarios el año 2011, lo que aumenta a 2,55 por 1.000 beneficiarios para el año 2012. Todo lo anterior comprueba que el número de estos pacientes está aumentando, lo que es concordante con los cambios sociodemográficos y epidemiológicos que enfrenta el país; la duda que cabe es si existen barreras de acceso y si existe la capacidad de oferta para atender digna y oportunamente a estos pacientes. 
TABLA N ${ }^{\circ} 2$

Distribución numérica y porcentual de la Discapacidad Severa bajo control según sexo, SS y País, años 2011 - 2012.

\begin{tabular}{|c|c|c|c|c|c|c|c|c|}
\hline & \multicolumn{4}{|c|}{2011} & \multicolumn{4}{|c|}{2012} \\
\hline & \multicolumn{2}{|c|}{ Hombres } & \multicolumn{2}{|c|}{ Mujeres } & \multicolumn{2}{|c|}{ Hombres } & \multicolumn{2}{|c|}{ Mujeres } \\
\hline & $\mathrm{N}^{\circ}$ & $\%$ & $\mathrm{~N}^{\circ}$ & $\%$ & $\mathrm{~N}^{\circ}$ & $\%$ & $\mathrm{~N}^{\circ}$ & $\%$ \\
\hline País & 10.124 & $39 \%$ & 15.930 & $61 \%$ & 12.124 & $40 \%$ & 18.074 & $60 \%$ \\
\hline Arica & 101 & $40 \%$ & 154 & $60 \%$ & 140 & $41 \%$ & 204 & $59 \%$ \\
\hline Iquique & 94 & $35 \%$ & 174 & $65 \%$ & 141 & $39 \%$ & 225 & $61 \%$ \\
\hline Antofagasta & 162 & $42 \%$ & 222 & $58 \%$ & 280 & $40 \%$ & 423 & $60 \%$ \\
\hline Atacama & 105 & $39 \%$ & 165 & $61 \%$ & 55 & $38 \%$ & 88 & $62 \%$ \\
\hline Coquimbo & 496 & $36 \%$ & 885 & $64 \%$ & 483 & $37 \%$ & 813 & $63 \%$ \\
\hline Valparaíso San Antonio & 425 & $37 \%$ & 735 & $63 \%$ & 397 & $36 \%$ & 693 & $64 \%$ \\
\hline Viña del Mar Quillota & 498 & $36 \%$ & 868 & $64 \%$ & 432 & $37 \%$ & 744 & $63 \%$ \\
\hline Aconcagua & 119 & $38 \%$ & 194 & $62 \%$ & 155 & $37 \%$ & 267 & $63 \%$ \\
\hline Metropolitano Norte & 259 & $36 \%$ & 463 & $64 \%$ & 433 & $39 \%$ & 676 & $61 \%$ \\
\hline Metropolitano Occidente & 598 & $40 \%$ & 890 & $60 \%$ & 701 & $40 \%$ & 1.055 & $60 \%$ \\
\hline Metropolitano Central & 361 & $36 \%$ & 649 & $64 \%$ & 414 & $34 \%$ & 791 & $66 \%$ \\
\hline Metropolitano Oriente & 389 & $28 \%$ & 1.011 & $72 \%$ & 673 & $33 \%$ & 1.358 & $67 \%$ \\
\hline Metropolitano Sur & 854 & $36 \%$ & 1.512 & $64 \%$ & 924 & $39 \%$ & 1.416 & $61 \%$ \\
\hline Metropolitano Sur Oriente & 424 & $38 \%$ & 690 & $62 \%$ & 595 & $41 \%$ & 859 & $59 \%$ \\
\hline Del Libertador B. O’Higgins & 301 & $37 \%$ & 514 & $63 \%$ & 661 & $41 \%$ & 947 & $59 \%$ \\
\hline Del Maule & 580 & $44 \%$ & 753 & $56 \%$ & 785 & $42 \%$ & 1.103 & $58 \%$ \\
\hline Nuble & 700 & $43 \%$ & 923 & $57 \%$ & 613 & $43 \%$ & 806 & $57 \%$ \\
\hline Concepción & 474 & $38 \%$ & 777 & $62 \%$ & 533 & $39 \%$ & 851 & $61 \%$ \\
\hline Arauco & 152 & $45 \%$ & 188 & $55 \%$ & 132 & $51 \%$ & 126 & $49 \%$ \\
\hline Talcahuano & 286 & $41 \%$ & 411 & $59 \%$ & 296 & $42 \%$ & 414 & $58 \%$ \\
\hline Biobío & 519 & $47 \%$ & 588 & $53 \%$ & 491 & $44 \%$ & 636 & $56 \%$ \\
\hline Araucanía Norte & 175 & $43 \%$ & 234 & $57 \%$ & 250 & $47 \%$ & 278 & $53 \%$ \\
\hline Araucanía Sur & 777 & $38 \%$ & 1.251 & $62 \%$ & 1.009 & $44 \%$ & 1.262 & $56 \%$ \\
\hline Valdivia & 326 & $44 \%$ & 423 & $56 \%$ & 395 & $42 \%$ & 537 & $58 \%$ \\
\hline Osorno & 206 & $43 \%$ & 272 & $57 \%$ & 272 & $46 \%$ & 318 & $54 \%$ \\
\hline Del Reloncaví & 424 & $45 \%$ & 511 & $55 \%$ & 498 & $43 \%$ & 665 & $57 \%$ \\
\hline Chiloé & 182 & $38 \%$ & 295 & $62 \%$ & 232 & $39 \%$ & 370 & $61 \%$ \\
\hline Aysén & 29 & $48 \%$ & 31 & $52 \%$ & 46 & $46 \%$ & 54 & $54 \%$ \\
\hline Magallanes & 108 & $42 \%$ & 147 & $58 \%$ & 88 & $48 \%$ & 95 & $52 \%$ \\
\hline
\end{tabular}

Fuente: DEIS - MINSAL, Chile. 
La discapacidad severa según sexo tiene una distribución muy homogénea para los años 2011 y 2012, donde sobre el $60 \%$ de los pacientes bajo control son mujeres. Llama la atención que el SS Arauco presenta una disminución por debajo del $50 \%$ para el año 2012, así como los SS de Araucanía Norte y Magallanes muestran una tendencia similar para el mismo año. Los datos son concordantes con los niveles de consulta en el nivel primario, donde las mujeres consultan más que los hombres. Sin embargo sabiendo que el adulto mayor se enfrenta a un mayor riesgo de fragilidad conforme aumenta su edad, aquí también puede estar influyendo tanto en la mayor tasa de sobrevida de las mujeres, como el nivel de osteoporosis que le afecta más en casos de accidentes y caídas.

TABLA No 3

Distribución numérica y porcentual de la Discapacidad Severa bajo control según grupos de edad, SS y País, años $2011-2012$

\begin{tabular}{|c|c|c|c|c|c|c|c|c|c|c|c|}
\hline & \multicolumn{11}{|c|}{2011} \\
\hline & \multicolumn{2}{|c|}{$<10$ años } & \multicolumn{2}{|c|}{10 a 19 años } & \multicolumn{2}{|c|}{20 a 44 años } & \multicolumn{2}{|c|}{45 a 64 años } & \multirow{2}{*}{$\frac{65 y+}{N^{\circ}}$} & \multirow[b]{2}{*}{$\%$} & \multirow[b]{2}{*}{ TOTAL } \\
\hline & $\mathrm{N}^{\circ}$ & $\%$ & $N^{\circ}$ & $\%$ & $\mathrm{~N}^{\circ}$ & $\%$ & $N^{\circ}$ & $\%$ & & & \\
\hline País & 804 & $3 \%$ & 1.336 & $5 \%$ & 2.556 & $10 \%$ & 2.605 & $10 \%$ & 18.753 & $72 \%$ & 26.054 \\
\hline Arica & 10 & $4 \%$ & 20 & $8 \%$ & 22 & $9 \%$ & 26 & $10 \%$ & 177 & $69 \%$ & 255 \\
\hline Iquique & 5 & $2 \%$ & 15 & $6 \%$ & 15 & $6 \%$ & 22 & $8 \%$ & 211 & $79 \%$ & 268 \\
\hline Antofagasta & 9 & $2 \%$ & 33 & $9 \%$ & 59 & $15 \%$ & 49 & $13 \%$ & 234 & $61 \%$ & 384 \\
\hline Atacama & 19 & $7 \%$ & 24 & $9 \%$ & 28 & $10 \%$ & 22 & $8 \%$ & 177 & $66 \%$ & 270 \\
\hline Coquimbo & 16 & $1 \%$ & 42 & $3 \%$ & 97 & $7 \%$ & 121 & $9 \%$ & 1.105 & $80 \%$ & 1.381 \\
\hline Valparaíso San Antonio & 22 & $2 \%$ & 34 & $3 \%$ & 88 & $8 \%$ & 121 & $10 \%$ & 895 & $77 \%$ & 1.160 \\
\hline Viña del Mar Quillota & 25 & $2 \%$ & 57 & $4 \%$ & 89 & $7 \%$ & 125 & $9 \%$ & 1.070 & $78 \%$ & 1.366 \\
\hline Aconcagua & 15 & $5 \%$ & 17 & $5 \%$ & 41 & $13 \%$ & 39 & $12 \%$ & 201 & $64 \%$ & 313 \\
\hline Metropolitano Norte & 15 & $2 \%$ & 27 & $4 \%$ & 78 & $11 \%$ & 91 & $13 \%$ & 511 & $71 \%$ & 722 \\
\hline Metropolitano Occidente & 74 & $5 \%$ & 93 & $6 \%$ & 197 & $13 \%$ & 185 & $12 \%$ & 939 & $63 \%$ & 1.488 \\
\hline Metropolitano Central & 16 & $2 \%$ & 28 & $3 \%$ & 81 & $8 \%$ & 101 & $10 \%$ & 784 & $78 \%$ & 1.010 \\
\hline Metropolitano Oriente & 12 & $1 \%$ & 32 & $2 \%$ & 74 & $5 \%$ & 121 & $9 \%$ & 1.161 & $83 \%$ & 1.400 \\
\hline Metropolitano Sur & 50 & $2 \%$ & 86 & $4 \%$ & 164 & $7 \%$ & 205 & $9 \%$ & 1.861 & $79 \%$ & 2.366 \\
\hline Metropolitano Sur Oriente & 29 & $3 \%$ & 64 & $6 \%$ & 145 & $13 \%$ & 120 & $11 \%$ & 756 & $68 \%$ & 1.114 \\
\hline Del Libertador B. O’Higgins & 18 & $2 \%$ & 40 & $5 \%$ & 61 & $7 \%$ & 74 & $9 \%$ & 622 & $76 \%$ & 815 \\
\hline Del Maule & 35 & $3 \%$ & 69 & $5 \%$ & 147 & $11 \%$ & 111 & $8 \%$ & 971 & $73 \%$ & 1.333 \\
\hline Ñuble & 46 & $3 \%$ & 88 & $5 \%$ & 192 & $12 \%$ & 187 & $12 \%$ & 1.110 & $68 \%$ & 1.623 \\
\hline Concepción & 57 & $5 \%$ & 94 & $8 \%$ & 131 & $10 \%$ & 136 & $11 \%$ & 833 & $67 \%$ & 1.251 \\
\hline Arauco & 20 & $6 \%$ & 27 & $8 \%$ & 35 & $10 \%$ & 39 & $11 \%$ & 219 & $64 \%$ & 340 \\
\hline Talcahuano & 39 & $6 \%$ & 51 & $7 \%$ & 94 & $13 \%$ & 74 & $11 \%$ & 439 & $63 \%$ & 697 \\
\hline Biobío & 52 & $5 \%$ & 73 & $7 \%$ & 122 & $11 \%$ & 96 & $9 \%$ & 764 & $69 \%$ & 1.107 \\
\hline Araucanía Norte & 27 & $7 \%$ & 30 & $7 \%$ & 63 & $15 \%$ & 49 & $12 \%$ & 240 & $59 \%$ & 409 \\
\hline Araucanía Sur & 92 & $5 \%$ & 114 & $6 \%$ & 200 & $10 \%$ & 195 & $10 \%$ & 1.427 & $70 \%$ & 2.028 \\
\hline Valdivia & 34 & $5 \%$ & 31 & $4 \%$ & 93 & $12 \%$ & 74 & $10 \%$ & 517 & $69 \%$ & 749 \\
\hline Osorno & 16 & $3 \%$ & 36 & $8 \%$ & 51 & $11 \%$ & 40 & $8 \%$ & 335 & $70 \%$ & 478 \\
\hline
\end{tabular}




\begin{tabular}{|c|c|c|c|c|c|c|c|c|c|c|c|}
\cline { 2 - 12 } \multicolumn{1}{c|}{} & \multicolumn{10}{|c|}{2011} & \multicolumn{10}{|c|}{$\mid$} \\
\hline Del Reloncaví & 34 & $4 \%$ & 63 & $7 \%$ & 113 & $12 \%$ & 101 & $11 \%$ & 624 & $67 \%$ & 935 \\
\hline Chiloé & 15 & $3 \%$ & 25 & $5 \%$ & 52 & $11 \%$ & 37 & $8 \%$ & 348 & $73 \%$ & 477 \\
\hline Aisén & 0 & $0 \%$ & 7 & $12 \%$ & 7 & $12 \%$ & 7 & $12 \%$ & 39 & $65 \%$ & 60 \\
\hline Magallanes & 2 & $1 \%$ & 16 & $6 \%$ & 17 & $7 \%$ & 37 & $15 \%$ & 183 & $72 \%$ & 255 \\
\hline
\end{tabular}

Fuente: DEIS - MINSAL, Chile.

TABLA No 4

Distribución numérica y porcentual de la Discapacidad Severa bajo control según sexo, SS y País, años 2011 - 2011

\begin{tabular}{|c|c|c|c|c|c|c|c|c|c|c|c|}
\hline & & & & & 2012 & & & & & & \\
\hline & \multicolumn{2}{|c|}{$<10$ años } & \multicolumn{2}{|c|}{10 a 19 años } & \multicolumn{2}{|c|}{20 a 44 años } & \multicolumn{2}{|c|}{45 a 64 años } & \multicolumn{2}{|c|}{$65 y+$} & \\
\hline & $\mathrm{N}^{\circ}$ & $\%$ & $\mathrm{~N}^{\circ}$ & $\%$ & $\mathrm{~N}^{\circ}$ & $\%$ & $\mathrm{~N}^{\circ}$ & $\%$ & $\mathrm{~N}^{\circ}$ & $\%$ & TOTAL \\
\hline País & 878 & $3 \%$ & 1.510 & $5 \%$ & 3.060 & $10 \%$ & 2.909 & $10 \%$ & 21.841 & $72 \%$ & 30.198 \\
\hline Arica & 7 & $2 \%$ & 27 & $8 \%$ & 25 & $7 \%$ & 29 & $8 \%$ & 256 & $74 \%$ & 344 \\
\hline Iquique & 12 & $3 \%$ & 21 & $6 \%$ & 24 & $7 \%$ & 51 & $14 \%$ & 258 & $70 \%$ & 366 \\
\hline Antofagasta & 25 & $4 \%$ & 38 & $5 \%$ & 106 & $15 \%$ & 64 & $9 \%$ & 470 & $67 \%$ & 703 \\
\hline Atacama & 8 & $6 \%$ & 7 & $5 \%$ & 30 & $21 \%$ & 11 & $8 \%$ & 87 & $61 \%$ & 143 \\
\hline Coquimbo & 30 & $2 \%$ & 41 & $3 \%$ & 111 & $9 \%$ & 147 & $11 \%$ & 967 & $75 \%$ & 1.296 \\
\hline Valparaíso San Antonio & 25 & $2 \%$ & 46 & $4 \%$ & 99 & $9 \%$ & 110 & $10 \%$ & 810 & $74 \%$ & 1.090 \\
\hline Viña del Mar Quillota & 10 & $1 \%$ & 51 & $4 \%$ & 85 & $7 \%$ & 103 & $9 \%$ & 922 & $79 \%$ & 1.171 \\
\hline Aconcagua & 18 & $4 \%$ & 21 & $5 \%$ & 68 & $16 \%$ & 35 & $8 \%$ & 280 & $66 \%$ & 422 \\
\hline Metropolitano Norte & 12 & $1 \%$ & 32 & $3 \%$ & 67 & $6 \%$ & 128 & $12 \%$ & 870 & $78 \%$ & 1.109 \\
\hline Metropolitano Occidente & 71 & $4 \%$ & 114 & $6 \%$ & 199 & $11 \%$ & 174 & $10 \%$ & 1.198 & $68 \%$ & 1.756 \\
\hline Metropolitano Central & 27 & $2 \%$ & 45 & $4 \%$ & 102 & $8 \%$ & 97 & $8 \%$ & 934 & $78 \%$ & 1.205 \\
\hline Metropolitano Oriente & 15 & $1 \%$ & 38 & $2 \%$ & 110 & $5 \%$ & 159 & $8 \%$ & 1.709 & $84 \%$ & 2.031 \\
\hline Metropolitano Sur & 71 & $3 \%$ & 94 & $4 \%$ & 218 & $9 \%$ & 222 & $9 \%$ & 1.735 & $74 \%$ & 2.340 \\
\hline Metropolitano Sur Oriente & 55 & $4 \%$ & 85 & $6 \%$ & 172 & $12 \%$ & 196 & $13 \%$ & 946 & $65 \%$ & 1.454 \\
\hline Del Libertador B. O’Higgins & 41 & $3 \%$ & 75 & $5 \%$ & 145 & $9 \%$ & 123 & $8 \%$ & 1.224 & $76 \%$ & 1.608 \\
\hline Del Maule & 50 & $3 \%$ & 96 & $5 \%$ & 192 & $10 \%$ & 168 & $9 \%$ & 1.382 & $73 \%$ & 1.888 \\
\hline Ñuble & 47 & $3 \%$ & 75 & $5 \%$ & 190 & $13 \%$ & 144 & $10 \%$ & 963 & $68 \%$ & 1.419 \\
\hline Concepción & 54 & $4 \%$ & 108 & $8 \%$ & 155 & $11 \%$ & 138 & $10 \%$ & 929 & $67 \%$ & 1.384 \\
\hline Arauco & 14 & $5 \%$ & 27 & $10 \%$ & 28 & $11 \%$ & 33 & $13 \%$ & 156 & $60 \%$ & 258 \\
\hline Talcahuano & 36 & $5 \%$ & 35 & $5 \%$ & 94 & $13 \%$ & 69 & $10 \%$ & 476 & $67 \%$ & 710 \\
\hline Biobío & 53 & $5 \%$ & 75 & $7 \%$ & 141 & $13 \%$ & 98 & $9 \%$ & 760 & $67 \%$ & 1.127 \\
\hline Araucanía Norte & 17 & $3 \%$ & 39 & $7 \%$ & 67 & $13 \%$ & 53 & $10 \%$ & 352 & $67 \%$ & 528 \\
\hline Araucanía Sur & 66 & $3 \%$ & 111 & $5 \%$ & 220 & $10 \%$ & 196 & $9 \%$ & 1.678 & $74 \%$ & 2.271 \\
\hline Valdivia & 37 & $4 \%$ & 53 & $6 \%$ & 100 & $11 \%$ & 81 & $9 \%$ & 661 & $71 \%$ & 932 \\
\hline
\end{tabular}




\begin{tabular}{|c|c|c|c|c|c|c|c|c|c|c|c|}
\hline & & & & & 2012 & & & & & & \\
\hline Osorno & 16 & $3 \%$ & 47 & $8 \%$ & 61 & $10 \%$ & 57 & $10 \%$ & 409 & $69 \%$ & 590 \\
\hline Del Reloncaví & 41 & $4 \%$ & 58 & $5 \%$ & 159 & $14 \%$ & 143 & $12 \%$ & 762 & $66 \%$ & 1.163 \\
\hline Chiloé & 15 & $2 \%$ & 35 & $6 \%$ & 63 & $10 \%$ & 40 & $7 \%$ & 449 & $75 \%$ & 602 \\
\hline Aisén & 3 & $3 \%$ & 8 & $8 \%$ & 9 & $9 \%$ & 15 & $15 \%$ & 65 & $65 \%$ & 100 \\
\hline Magallanes & 2 & $1 \%$ & 8 & $4 \%$ & 15 & $8 \%$ & 25 & $14 \%$ & 133 & $73 \%$ & 183 \\
\hline
\end{tabular}

Fuente: DEIS - MINSAL, Chile.

Al analizar los datos de las tablas 3 y 4 , se observa que según grupos de edad; la discapacidad severa controlada en los Centros o CESFAM del país aumenta conforme aumenta la edad de los pacientes bajo control. Se visualiza que un $65 \%$ de los pacientes con discapacidad severa controlados en los centros de Atención Primaria del país son mayores de 65 años, existiendo grandes diferencias entre los grupos de niños y adoles- centes, así como tampoco entre adultos jóvenes y adultos de edad media. Cabe señalar que la distribución etaria encontrada en la discapacidad no es coincidente con la distribución etaria del país, ya que en este último caso la población se concentra en el grupo de adultos que conforman el mayor volumen, no obstante la discapacidad se concentra en los adultos mayores.

\section{FIGURA $N^{\circ} 1$}

Tasas de Discapacidad Severa por grupos de edad en población beneficiaria FONASA, Chile, años 2011 - 2012

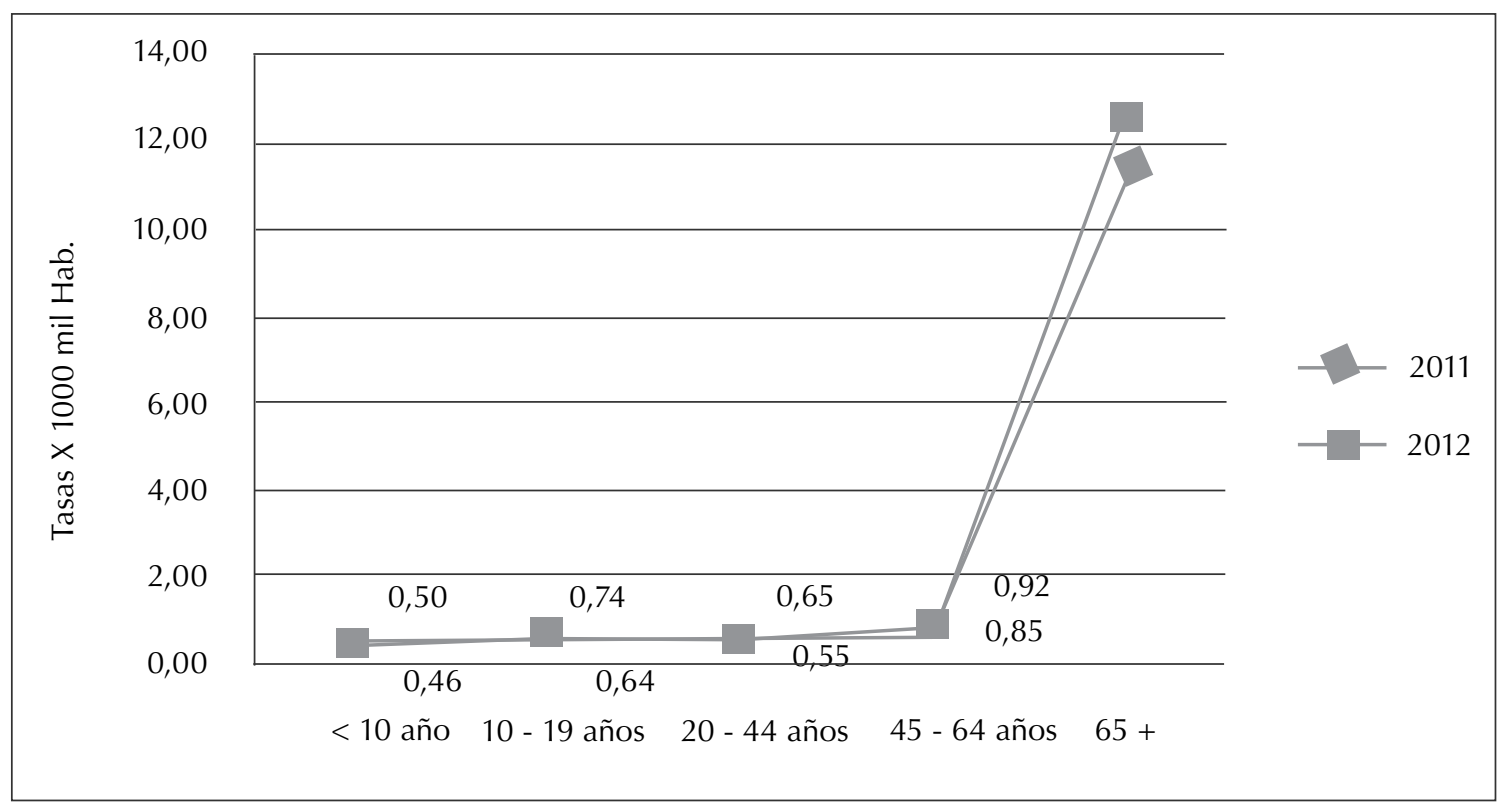

Fuente: DEIS - MINSAL, Chile. 
Las tasas de discapacidad severa en población bajo control por mil habitantes en nuestro país aumentan conforme aumenta la edad, siendo muy notorio en las personas mayores de 65 años. Existen tasas significativamente mayores en los diferentes grupos de edad el año 2012 respecto del 2011. Lo anterior es concordante con los datos del censo del año 2002 y con el Estudio Nacional de Discapacidad realizado el año 2005 (La Discapacidad en Chile, Ministerio de Planificación, 2006).

TABLA N ${ }^{\circ} 5$

Tasas de Discapacidad Severa en población beneficiaria FONASA bajo control según sexo, SS y País, años 2011 - 2012

\begin{tabular}{|c|c|c|c|c|c|c|}
\hline \multirow{2}{*}{ Servicio de Salud } & Tasas & $2011^{*}$ & & Tasas & $2012 *$ & \\
\hline & Mujer & Hombre & Total & Mujer & Hombre & Total \\
\hline Arica & 1,7 & 1,3 & 1,5 & 2,3 & 1,7 & 2,0 \\
\hline Iquique & 1,5 & 0,9 & 1,2 & 1,9 & 1,3 & 1,6 \\
\hline Antofagasta & 1,2 & 1,0 & 1,1 & 2,2 & 1,6 & 1,9 \\
\hline Atacama & 1,4 & 0,9 & 1,2 & 0,7 & 0,5 & 0,6 \\
\hline Coquimbo & 3,0 & 1,8 & 2,4 & 2,7 & 1,7 & 2,2 \\
\hline Valparaíso San Antonio & 3,4 & 2,2 & 2,9 & 3,2 & 2,1 & 2,7 \\
\hline Viña del Mar Quillota & 2,1 & 1,4 & 1,8 & 1,8 & 1,2 & 1,5 \\
\hline Aconcagua & 1,7 & 1,1 & 1,5 & 2,4 & 1,5 & 2,0 \\
\hline Metropolitano Norte & 1,4 & 0,9 & 1,2 & 2,0 & 1,4 & 1,7 \\
\hline Metropolitano Occidente & 1,7 & 1,3 & 1,5 & 2,1 & 1,5 & 1,8 \\
\hline Metropolitano Central & 1,9 & 1,3 & 1,6 & 2,0 & 1,3 & 1,7 \\
\hline Metropolitano Oriente & 2,8 & 1,5 & 2,3 & 3,9 & 2,7 & 3,4 \\
\hline Metropolitano Sur & 3,1 & 2,0 & 2,6 & 2,9 & 2,1 & 2,5 \\
\hline Metropolitano Sur Oriente & 1,2 & 0,8 & 1,0 & 1,5 & 1,2 & 1,3 \\
\hline Libertador B. O'Higgins & 1,5 & 0,9 & 1,2 & 2,6 & 1,9 & 2,3 \\
\hline Maule & 1,7 & 1,4 & 1,5 & 2,5 & 1,9 & 2,2 \\
\hline Ñuble & 4,3 & 3,5 & 3,9 & 3,8 & 3,1 & 3,5 \\
\hline Concepción & 3,1 & 2,1 & 2,6 & 3,3 & 2,3 & 2,9 \\
\hline Arauco & 2,5 & 2,1 & 2,3 & 1,6 & 1,8 & 1,7 \\
\hline Talcahuano & 2,6 & 2,0 & 2,4 & 2,6 & 2,1 & 2,4 \\
\hline Biobío Los Angeles & 3,4 & 3,2 & 3,3 & 3,7 & 3,0 & 3,3 \\
\hline Araucanía Norte & 2,6 & 2,1 & 2,3 & 3,1 & 3,0 & 3,0 \\
\hline Araucanía Sur & 4,0 & 2,7 & 3,4 & 4,0 & 3,5 & 3,8 \\
\hline Valdivia & 2,5 & 2,1 & 2,3 & 3,3 & 2,6 & 2,9 \\
\hline Osorno & 2,6 & 2,1 & 2,4 & 3,0 & 2,8 & 2,9 \\
\hline Del Reloncaví & 2,9 & 2,6 & 2,7 & 4,0 & 3,2 & 3,6 \\
\hline Chiloé & 3,9 & 2,6 & 3,3 & 4,8 & 3,3 & 4,1 \\
\hline Aysén & 0,8 & 0,8 & 0,8 & 1,4 & 1,2 & 1,3 \\
\hline Magallanes & 2,5 & 2,0 & 2,2 & 1,5 & 1,5 & 1,5 \\
\hline Total & 2,3 & 1,6 & 2,0 & 2,6 & 1,9 & 2,3 \\
\hline
\end{tabular}

Fuente: DEIS - MINSAL, Chile. * Tasas por 1.000 Habitantes. 
Al evaluar las tasas de la población con discapacidad severa bajo control en relación a población beneficiaria FONASA, se aprecia que la tasa nacional aumenta desde el año 2011 al 2012, lo que se repite por y Servicio Social. Se observa que los SS de los extremos norte y sur muestran tasas por debajo de la tasa nacional, Ilamando la atención la baja tasa del SS de Atacama el año 2012, donde pudiera influir un posible sub registro de los datos para este año. En la Región Metropolitana destaca el SS Metropolitano Oriente, que es coincidente con ser una de las comunas con un alto nivel de envejecimiento en el país. A la vez, las tasas más altas están en los SS de las zonas centro sur y sur del país, destacando los SS de Chiloé, Araucanía Sur, Del Reloncaví, Ñuble, Concepción, Valdivia y Osorno, todo lo cual es coincidente con el Estudio Nacional de Dependencia del año 2010 que muestra que las regiones VIII, IX y X están dentro de las regiones con mas discapacidad en el país (Estudio Nacional de la Dependencia en Adultos Mayores, 2010) y con el primer Estudio Nacional de Discapacidad que muestra qué en zonas con más ruralidad hay un aumento de la discapacidad (Primer Estudio Nacional de Discapacidad, 2005).

\section{Discusion}

Del análisis de las tablas destaca el incremento de pacientes con discapacidad severa el año 2012 respecto del 2011, predominando el sexo femenino. Sobresale el aumento exponencial después de los 65 años, donde la tasa de discapacidad severa por 1.000 habitantes adscritos a FONASA es 10 a 11 veces mayor que otros grupos de edad, lo que se suma al aumento de la prevalencia de enfermedades crónicas en los mayores de 60 años $^{7}$, así como las secuelas de enfermedades cardiovasculares y las discapacidades de tipo cognitivo. De acuerdo a la bibliografía revisada, todos hacen el corte de adulto mayor a los 60 años, no obstante el DEIS - MINSAL mantiene en sus REM el corte a los 65 años, por lo que se sugiere cambiarlo.

El Estudio de Discapacidad en Chile del año 2006 mostró que el $55 \%$ de la discapacidad era física, un $28 \%$ auditiva y visual, y un $17 \%$ psíquica e intelectual,

La encuesta nacional de Dependencia del año 2010 demostró que el $68 \%$ de las personas mayores de 60 años en el país tienen entre 1 a 3 enfermedades crónicas y un $6,8 \%$ entre 4 a 6 . de los cuales sólo el 6,8\% refirió acceder a programas de rehabilitación y donde los adultos mayores tenían el más bajo acceso. La prevalencia de discapacidad severa encontrada en la encuesta nacional del año 2005 fue de 2,5\%, que aplicada a los pacientes adscritos a FONASA el año 2012 arroja 334.427 personas, con lo que se infiere que la cobertura de pacientes bajo control para el mismo año en nuestros centros es de un $9 \%$, lo que demuestra que no estamos Ilegando a este grupo social altamente vulnerable.

Sería interesante confirmar si en las comunas extremas hay menos pacientes bajo control o existe una falta de pesquisa o de acceso a la atención por falta de recursos; a la vez, sería interesante estudiar qué factores influyen en la concentración de la discapacidad severa en los SS de la zona sur del país y evaluar si existe alguna correlación entre nuestro hallazgo y el deterioro de algunos indicadores sanitarios y de atención en estas zonas geográficas, tal como se aprecia en el estudio de la Organización Panamericana de la Salud (OPS) el año 2010 (OPS, 2010).

Este trabajo abre una importante línea de estudio en salud que permita caracterizar demográfica y epidemiológicamente, y según la forma de atención a estos pacientes, que sirva de base para redefinir políticas y focalizar recursos para este tipo de pacientes. En este sentido, según lo estudiado por OPS (OPS, 2010), existe una muy baja tasa por 10.000 habitantes de profesionales Terapeutas Ocupacionales en el Sistema Nacional de Servicios de Salud (0,1 x 10.000 habitantes), así como de otros profesionales, los que en conjunto son claves para la realización de actividades de Prevención y Promoción de la Salud en hombres y mujeres adultos para evitar el alto costo sanitario y social de la rehabilitación de los pacientes con dependencia severa en nuestro país.

La política de salud requiere una orientación urgente dirigida a prevenir la discapacidad en sus diferentes grados en los adultos mayores, más aún cuando la Ley $N^{\circ} 20.422$ de la Discapacidad enfatiza en su Título III, art. $18^{\circ}, 19^{\circ}, 20^{\circ}$ y $21^{\circ}$ la prevención y rehabilitación de la discapacidad. Ya no es discutible que en nuestro país el adulto mayor se enfrenta a un sistema económico y social altamente regresivo, producto del empobrecimiento que vive a medida que sigue envejeciendo, lo que es más grave en pacientes con discapacidad severa y estados de postración severa. Por ello, las acciones de Promoción y Prevención de la Salud debieran comenzar en las primeras etapas de la vida del adulto y adulto maduro, promoviendo en estos grupos estilos 
de vida saludables con enfoque intersectorial y de red, para impactar en las condicionantes sociales y evitar llegar tardíamente a este grupo de pacientes, más aún con el perfil de envejecimiento acelerado que enfrenta nuestro país.

\section{RefERENCIAS BibliOgRÁfICAS}

BID/CEPAL/CELADE (2000). La transición demográfica en América Latina.

Dorentes G., Ávila J., Mejía S., Gutiérrez L. (2001). Factores asociados con la dependencia funcional en los adultos mayores: Un análisis secundario del Estudio Nacional sobre Salud y Envejecimiento en México, Revista Panamericana de Salud Pública, 2001.

Barthel D., Mahoney F. (1965). Funtional evaluation: Barthel Index. Md State Med J.; 14:61-5.

Baltes M., Wahl H., Editors (1990). Dependencia en los ancianos. Barcelona: Editorial Martínez-Roca.

IMERSO (2007). Libro Blanco de la Dependencia, Madrid.

Abanto A. (2010). Demografía del paciente crónico: Medicina Geriátrica y Residencias. EDIMSA, Madrid.

Clasificación Internacional de Deficiencias, Discapacidades y Minusvalías (CIDDM). (1980).

Querejeta M. (2004). Discapacidad/Dependencia: Unificación de criterios de valoración y clasificación, Ministerio del Trabajo y Asuntos Sociales: Secretaría de Estado de Servicios Sociales, Familias y Discapacidad. IMERSO, Madrid.

Observatorio Demográfico parea América Latina y el Caribe, Proyecciones de Población (2012).

INE (2008). Proyecciones y Estimaciones de Población. 1990-2020. País y Regiones; http://www.ine.cl/canales/chile_estadistico/ demografia_y_vitales/proyecciones/INFORME/informe_proyecciones_2008.pdf.

Calvo A., Tartakowsky A., Maffei T. (2001). Transformaciones en las estructuras familiares en Chile, MIDEPLAN.

Estudio Nacional de Dependencia en Personas Mayores, Servicio Nacional del Adulto Mayor (2010).

Quiroga P., Albala C., Klaasen G. (2004). Validation of a screening test for age associated cognitive impairment, in Chile. Revista Médica de Chile; 132(4):467-78.

Katz S., Ford A., Moskowitz R., Jackson B., Jaffe M. (1963). Studies of Illness in the Aged. The Index of Adl: A Standardized Measure of Biological and Psychosocial Function. JAMA, 21;185:914-9.

Lawton M., Brody E. (1969). Assessment of older people: Selfmaintaining and instrumental activities of daily living. Gerontologist; 9(3): 179-86.
Norma de cuidados domiciliarios de personas que sufren Discapacidad Severa (2006). División de Prevención y Control de Enfermedades, Depto. Ciclo Vital Programa del Adulto Mayor, Unidad de Discapacidad y Rehabilitación, MINSAL.

Depto. de Planificación Institucional, Sub Depto. de Estudios. (2012) FONASA.

La Discapacidad en Chile (2006). Paso hacia un Modelo Integral de funcionamiento humano, Fondo Nacional de la Discapacidad, Ministerio de Planificación, Chile.

Estudio Nacional de la Dependencia en Adultos Mayores (2010). SENAMA.

Primer Estudio Nacional de Discapacidad, Fondo Nacional de la Discapacidad (2005). Gobierno de Chile.

Salud en Chile, Panorama de la Situación de Salud y del Sistema de Salud en Chile (2010). OPS. 